\title{
SOME THEOREMS CONCERNING THE CONTINUITY OF ALGEBRA HOMOMORPHISMS ${ }^{1}$
}

\author{
BRUCE ALAN BARNES
}

In this note we establish a useful property of the separating set of an algebra homomorphism of one Banach algebra into another (Theorem 1), and we use this result to prove two theorems concerning the continuity of algebra homomorphisms (Theorems 2 and 3). We assume throughout that $A$ and $B$ are complex Banach algebras with norms $\|\cdot\|_{A}$ and $\|\cdot\|_{B}$ respectively, and that $\nu$ is an algebra homomorphism of $B$ into $A$. By the separating set of $\nu$, we mean the set of all $u \in A$ such that there exists a sequence $\left\{v_{n}\right\}$ in $B$ with the property that $\left\|v_{n}\right\|_{B} \rightarrow 0$ and $\left\|\nu\left(v_{n}\right)-u\right\|_{A} \rightarrow 0$. We denote this set by $\mathcal{S}$. $\nu$ is continuous if and only if $\delta=0$ by the Closed Graph Theorem. If $C$ is any algebra and $u \in C$, then $\sigma_{C}(u)$ is the spectrum of $u$ in $C$, and

$$
\rho_{C}(u)=\sup \left\{|\lambda| \mid \lambda \in \sigma_{C}(u)\right\} .
$$

The first theorem we prove is a direct application of a result of J. D. Newburgh. Assume that $u \in A$, and that $E$ is a nonempty open and closed subset of $\sigma_{A}(u)$. Let $V$ be an open neighborhood of $E$. If $\left\{u_{n}\right\}$ is a sequence in $A$ such that $u_{n} \rightarrow u$, then Newburgh proved that there exists an integer $N$ such that $n \geqq N$ implies that $\sigma_{A}\left(u_{n}\right) \cap V$ is nonempty; see [5, Lemma 3, p. 168]. Now suppose that $u \in \mathcal{S}$, and that $u$ has disconnected spectrum in $A$. Then there exists a nonempty open and closed subset $E$ of $\sigma_{A}(u)$ such that $0 \notin E$. Therefore we can choose a neighborhood $V$ of $E$ whose closure does not contain 0 . Now since $u \in \mathcal{S}$, there exists $\left\{v_{n}\right\} \subset B$ such that $\left\|v_{n}\right\|_{B} \rightarrow 0$ and $\left\|\nu\left(v_{n}\right)-u\right\|_{A} \rightarrow 0$. It follows from Newburgh's result that there exists $\alpha>0$ such that $\rho_{A}\left(\nu\left(v_{n}\right)\right)>\alpha$ for $n$ large enough. But

$$
\rho_{A}\left(\nu\left(v_{n}\right)\right) \leqq \rho_{\nu(B)}\left(\nu\left(v_{n}\right)\right) \leqq \rho_{B}\left(v_{n}\right) \leqq\left\|v_{n}\right\|_{B},
$$

and $\left\|v_{n}\right\|_{B} \rightarrow 0$. We have reached a contradiction, and this proves the following theorem.

Theorem 1. S contains no elements of $A$ which have disconnected spectrum in $A$. In particular $S$ contains no nonzero idempotents.

Next we make an immediate application of Theorem 1 . Here $S$

Received by the editors September 17, 1966.

1 This research was supported by National Science Foundation Grant number GP-5585. 
denotes the socle of $A$, and $L[S]=\{a \in A \mid a S=0\}$.

Theorem 2. Assume that $A$ and $B$ are complex Banach algebras and that $\nu(B)$ is dense in $A$. Assume also that $A$ is semisimple. Then $S \subset L[S]$. In particular if $L[S]=0$ then $\nu$ is continuous.

Proof. Since $\nu(B)$ is dense in $A$, it follows by [7, Lemma 3.1 , p. 377], that $\delta$ is an ideal of $A$. Now either $\delta \subset L[S]$ or $\delta$ contains a minimal left ideal by [1, Proposition 3.3, p. 567]. But $\delta$ can contain no idempotents, and in particular, $\delta$ contains no minimal idempotent. Therefore $\delta$ must be included in $L[S]$.

Many important algebras have the property that the left annihilator of their socle is zero. In particular, any semisimple modular annihilator algebra has this property by [1, Theorem 4.2 (2), p. 569]. We state this particular result as a corollary.

Corollary. Assume that $A$ and $B$ are complex Banach algebras and that $\nu(B)$ is dense in $A$. Furthermore assume that $A$ is a semisimple modular annihilator algebra. Then $\nu$ is continuous.

In the case where $A$ is a strongly semisimple modular annihilator algebra, and $B$ satisfies a certain special condition, we can drop the assumption of the Corollary that $\nu(B)$ be dense in $A$. First we state the condition we impose on $B$.

$\left.{ }^{*}\right)$ Whenever $I$ is a closed ideal of $B$ such that $B / I$ is finite dimensional, then $I^{2}=I$.

We shall prove the following theorem.

Theorem 3. Assume that $B$ is a complex Banach algebra which satisfies condition $\left({ }^{*}\right)$, and assume that $A$ is a complex, strongly semisimple modular annihilator algebra. Then $\nu$ is continuous.

Before proving this theorem, we note some examples of algebras $A$ and $B$ which satisfy its hypotheses. By a Theorem of P. J. Cohen, whenever a Banach algebra $B$ has a bounded approximate identity, then $B^{2}=B$; see $\left[2\right.$, Theorem 1 , p. 199]. Now since any $B^{*}$-algebra has a bounded approximate identity by [6, Theorem (4.8.14), p. 245], and since every closed ideal $I$ of a $B^{*}$-algebra is again a $B^{*}$-algebra by $[6$, Theorem $(4.9 .2)$, p. 249$]$, then automatically $I^{2}=I$. Therefore every $B^{*}$-algebra satisfies condition $\left({ }^{*}\right)$. Assume now that $B$ is a dual algebra with a bounded approximate identity, that $I$ is a closed ideal of $B$, and that $B / I$ is finite dimensional. Since $I$ is a closed ideal of $B$, then $I$ is the intersection of primitive ideals of $B$ by $[1$, Theorem 5.1, p. 570$]$. It follows from [1, Theorem 6.5, p. 574], 
that each of these primitive ideals has the form $B(1-e)$ where $e$ is a central idempotent of $B$. Also $I$ is contained in only a finite number of primitive ideals of $B$. Then it can be shown that $I$ itself is of the form $B(1-e)$, where $e$ is a central idempotent of $B$. By assumption $B$ has a bounded approximate identity, and therefore $B=B^{2}$. Thus $I^{2}=B(1-e) \cdot B(1-e)=B^{2}(1-e)=I$. Therefore $B$ satisfies condition $\left({ }^{*}\right)$. We note that if $G$ is a compact group, then $L^{1}[G]$ is a dual algebra with a bounded approximate identity.

Examples of strongly semisimple modular annihilator algebras are $L^{p}[G]$ where $G$ is a compact group and $1 \leqq p \leqq \infty$ (see $[4$, Theorem 15 , p. 699]), and more generally, any semisimple completely continuous Banach algebra (see [1, Theorem 7.2, p. 576], and [1, Theorem 7.1, p. 575$])$.

-We begin the proof of Theorem 4 with a lemma.

Lemma. Assume that $B$ is a complex Banach algebra with property $\left.{ }^{*}\right)$. Assume that $A$ is complex and finite dimensional. Then $\nu$ is continuous.

Proof. Since $\nu(B)$ is finite dimensional, $\nu(B)$ is a closed subalgebra of $A$ and $\mathrm{S}$ is an ideal of $\nu(B)$. Also $\nu(B)$ satisfies the minimum condition; see $[3$, p. 38]. Then since $\delta$ contains no idempotents, $S$ is contained in the radical of $\nu(B)$. Now the radical of a ring with minimum condition is nilpotent by $[3$, Theorem 1, p. 38$]$, and it follows that there exists an integer $n$ such that $\delta^{n}=0$. Then if $I=\nu^{-1}(s), I$ is a closed ideal of $B$ by $[7$, Lemma 3.1, p. 377]. But then $I^{n}$ is contained in the kernel of $\nu$. Since $I^{n}=I$ by hypothesis, then $\delta=0$ and $\nu$ is continuous.

Now we complete the proof of Theorem 3. Let $A$ and $B$ be as in the hypotheses of Theorem 3. If $M$ is any maximal modular ideal of $A$, then by [1, Theorem 6.4 , p. 574], $A / M$ is finite dimensional. Let $\pi$ denote the natural projection of $A$ onto $A / M$. Then $\pi \nu$, the composition of $\pi$ with $\nu$, is an algebra homomorphism of $B$ into the finite dimensional algebra $A / M$. By the Lemma, $\pi \nu$ is continuous, and this implies that $S \subset M$. Now by assumption $A$ is strongly semisimple, that is the intersection of all the maximal modular ideals of $A$ is 0 . But $S$ is contained in this intersection by the argument above, and therefore $\delta=0$ and $\nu$ is continuous.

\section{REFERENCES}

1. B. A. Barnes, Modular annihilator algebras, Canad. J. Math. 18 (1966), 566578. 
2. P. J. Cohen, Factorization in group algebras, Duke Math. J. 26 (1959), 199-205.

3. N. Jacobson, Structure of rings, Colloq. Publ., Vol. 37, Amer. Math. Soc., Providence, R. I., 1956.

4. I. Kaplansky, Dual rings, Ann. of Math. 49 (1948), 689-701.

5. J. D. Newburgh, The variation of spectra, Duke Math. J. 18 (1951), 165-176.

6. C. E. Rickart, Banach algebras, Van Nostrand, Princeton, N. J., 1960.

7. B. Yood, Homomorphisms on normed algebras, Pacific J. Math. 8 (1958), 373381.

The University of California, Berkeley and

The UNIVERSITY OF Oregon

\section{GROUPS AND SEMIGROUPS WITH SOLVABLE WORD PROBLEMS}

B. H. MAYOH

This note gives a direct proof of the following theorem of M. O. Rabin [1, Theorem 4]:

THEOREM. A finitely generated group has a solvable word problem (with respect to a given system of generators) if and only if it is computable.

A group is said [1] to have a solvable word problem with respect to a finite set of generators $\left\{g_{1}, \cdots, g_{n}\right\}$ if one can effectively determine whether or not two words on $\left\{\sigma_{1}, \cdots, \sigma_{n}\right\}$ represent the same group element. (Rabin's definition, though equivalent for groups, prevents the theorem from applying to semigroups. For convenience we suppose that the inverse of a generator is a generator.) A group is said to be computable if it has a recursive realization $\{S, x\}$-i.e. it is isomorphic to the group formed by a recursive subset $S$ of the positive integers and a recursive function $x(i, j)$ on $S$ that satisfies the group multiplication axioms.

Proof. Consider some recursive realization, $\{S, x\}$ of a given finitely generated computable group. Let $s_{i}$ be the element of $S$ corresponding to the generator $g_{i}$. For any word $W=\sigma_{a} \sigma_{b} \cdots \sigma_{z}$ one can effectively compute the in teger

$$
w=s_{a} \times s_{b} \times \cdots \times s_{z} .
$$

As two such words, $W$ and $W^{1}$, are equivalent if and only if $w=w^{1}$,

Received by the editors September 15, 1966. 